Bull. Mater. Sci., Vol. 4, Number 3, May 1982, pp. 261-266, (C) Printed in India.

\title{
Processing of ceramics and glasses in space
}

\author{
P RAMACHANDRARAO \\ School of Materials Science and Technology, Banaras Hindu University, \\ Varanasi 221005 , India \\ MS received 21 April 1980
}

\begin{abstract}
Possible experiments in space on ceramics, composites and inorganic glasses are listed. Advantages in processing these materials under microgravity conditions, anticipated effects and likely problems are discussed. Theoretical conclusions and experimental results to date are reviewed. It is suggested that experiments on metallic glasses in space could prove to be rewarding.
\end{abstract}

Keywords. Single crystal growth; metal-ceramic composites; ceramic-ceramic composites; metallic glasses,

\section{Introduction}

As in the case of all other materials, attempts at processing of ceramics and glasses in space rely on weightlessness, the absence of gravity-induced convection and the suppression of segregation due to density differences. Gravitational forces play no direct role in controlling chemical reactions; but these conditions in space play an indirect role by affecting the diffusion kinetics and the extent of contamination during melting and solidification. Accordingly, experiments conducted in rocket flights and those proposed for the Spacelab are aimed at studying: (i) crucible free melting for the production of ultraclean materials; (ii) obtaining high strength endless ceramic fibres; (iii) growth of single crystals; (iv) slip casting; (v) production of metal-ceramic and ceramic-ceramic composites; (vi) diffusion in glass forming melts; and (vii) glass formation.

Various investigators have earlier studied this problem (Sahm 1977; Ahlborn 1977; Kaye 1974; Seibert 1978a,b; Takahashi 1975; Deutscher 1976). Some of the advantages, anticipated effects and problems likely to be encountered during the processing of ceramics and glasses in space are discussed in this article.

\section{Crystalline ceramies and composites}

Experiments on semiconductor materials have shown that growth under microgravity conditions results in the production of defect-free and chemically homogepeous single crystals with superior properties. Similar results can be expected in the case of ceramic materials and some experiments utilising Czochralski technique, without crystal or crucible rotation, are envisaged. Under terrestrial 
conditions, fluid flow induced by Marangoni effect is negligible. However, when the surface tension and its temperature coefficient are large and there are composition gradients as well, Marangoni effect can become important. It plays an even more significant role under microgravity conditions where there may be no other competing fluid flow phenomena. Temperature gradient-induced Marangoni flow is commonly referred to as thermocapillary flow. Early theoretical studies on the floating zone method have shown that thermocapillary flow can be very important in the growth of oxide crystals (Chang and Wilcox 1975). Under terrestrial conditions, the larger geometries of Czochralski growth crucibles permit considerable buoyant convection and seed rotation also induces centrifugal pumping. Both these effects mask thermocapillary flow. Buoyancy-induced convection is proportional to the acceleration due to gravity and plays a much lesser role under microgravity conditions, resulting thus in the dominance of thermocapillary flow. Langlois (1980) investigated the importance of Marangoni convection in the growth of alumina crystals by Czachralski method under microgravity conditions. The analysis employs the time-dependent Navier-Stokes equations (Langlois 1979). Axial symmetry, lack of heating by viscous dissipation, Stefan $\rightarrow$ Boltzmann radiation and flat stress-free surface were assumed in the analysis. It was shown that even for a substance like alumina which has a very small temperature coefficient of surface tension, buoyant convection is insignificant as compared to thermocapillary flow. Meridional circulation is quite intense and once the steady state conditions are established, variation in microgravity or $g$-jitter appears to have little or no influence in altering the flow conditions. Langlois (1980) further observed that the relatively strong thermacapillary flow may not altogether be disadvantageous. It could substantially redistribute heat and prevent "flarring". As pointed out by Langlois, variations in the direction of microgravity could possibly have an important effect on the Czochralski flow, but, no theoretical analysis of its influence has so far been carried out.

Weightless conditions under microgravity enable containerless processing by melting and solidification, avoiding any contamination by reaction with the otherwise necessary crucible materials. Such experiments are of importance in the production of ultraclean refractory dielectric, electrical and optical materials. Reaction of ultra-high purity levitated elemental charges, such as, silicon, with gaseous phases like, oxygen or nitrogen, to yield high quality dielectrics are also envisaged. It is hoped that characterisation of such materials will help establish their limiting properties and aid in appropriate material selection for further development on earth (Deutscher 1976). Techniques like zone refining could also be carried out in space for the production of pure ceramic materials and some proposals have been made in this direction as well (Deutscher 1976).

Elimination of gravity-induced segregation is expected to help in the production of novel metal-ceramic and ceramic-ceramic composites. An important problem in the production of such composites arises from the need for a strong bond between the dispersed material and the matrix. Coating or chemical treatment of the phase to be dispersed have been frequently used for development of the necessary bond. Studies have shown that the wetting of the dispersed phase by liquid matrix is prevented by the presence of gaseous and other impurities at the interface (Kaye 1974). Experiments with aluminium-alumina, aluminium- 
silicon carbide and aluminium-sapphire indicate that use of high vacuum, high temperature, cleaning of the second phase surface by ion bombardment and vigorous stirring of the molten mixture help in providing good bond (Kaye 1974). Availability of high vacuum, with attendant advantages of containerless processing under non-segregating conditions, makes fabrication of composites under microgravity conditions an attractive possibility. One proposal is to produce aluminium castings with a high proportion of non-oriented carbon fibres (Kohler 1978). Uniform dispersion of carbon in aluminium is expected to result in composites with improved mechanical properties under multi-axial loading conditions, better heat resistance and machinability.

One of the important areas of interest in materials processing in space concerns shaping of materials under conditions of weightlessness (Walter 1977). Factors such as surface tension and viscosity of the melt and the existence of electromagnetic, accoustic, boundary plane and centrifugal forces influence the shape of the molten mass. These forces can be manipulated to yield a desired shape. Theoretical attempts are in progress to determine the conditions under which wires, plates, foils, spheres, lenses, cylinders, etc., can be manufactured in space. Some of the experiments proposed for Spacelab deal with the shaping of ceramics and the production of endless high strength ceramic fibres for use in composites production (Deutscher 1976).

An alternate route for the fabrication of composites is through slip casting. One of the proposals submitted for Spacelab has the object of slip casting ceramicceramic and ceramic-metal composites with significant grain size and density differences. Both drain and solid type slip castings require a low rate of settling on standing and this should be easily achieved along with suppression of segregation under microgravity conditions. Slip casting has the further advantage of economy of energy and space, since the experiments can be carried out at ambient temperatures (Deutscher 1976).

\section{Investigations on glasses}

Diffusion kinetics plays an important role in the manufacture of glass at various stages such as melting, crucible-melt interaction, homogenisation and colouring. In the molten state, diffusion is normally influenced by the presence of convective flow. Studies under microgravity conditions enable an assessment of the role of diffusion, unaided by convection, in glass forming and processing. Herr et al (1979) have investigated the ion-exchange process in the quasi-binary system $\mathrm{Na}_{2} \mathrm{O} .3 \mathrm{SiO}_{2} \rightarrow \mathrm{Rb}_{2} \mathrm{O}, 3 \mathrm{SiO}_{2}$ as part of the TEXUS-I rocket programme. Under the influence of normal gravitational field a study of the process is greatly affected by convection at temperatures above $1000^{\circ} \mathrm{C}$. To eliminate this problem, two cylinders $(12 \mathrm{~cm}$ dia and $10 \mathrm{~mm}$ height) of the constituent glasses were contacted at their polished surfaces and enveloped in platinum, prior to insertion into a molybdenum cartridge. Before the rocket flight the samples were slowly heated to above their glass transition temperatures and during the flight the middle of the sample was rapidly raised to about $1160^{\circ} \mathrm{C}$ for an effective period of $135 \mathrm{sec}$ during which reaction occurred under conditions of $10^{-4} \mathrm{~g}$. Analysis of the 


\section{$P$ Ramachandrarao}

diffusion profiles indicated that ion-exchange, unaffected by convection, occurs under microgravity conditions. The binary chemical interdiffusion coefficient was found to be concentration-dependent. The frequency factor $D_{\circ}$ and the activation energy $Q$ were found to be $5 \times 10^{-3} \mathrm{~cm}^{2} \mathrm{sec}^{-1}$ and $75 \mathrm{~kJ} \mathrm{~mol}^{-1}$ respectively, in the temperature range of 973 to $1433 \mathrm{~K}$. The results encouraged further experimentation along similar lines.

The advent of techniques for the rapid solidification of metals and alloys at coaling rates above $10^{6} \mathrm{~K} \mathrm{sec}^{-1}$ has resulted in the development of a new and exotic class of materials, viz., metallic glasses. The interest in these materials stems fram their excellent mechanical, magnetic and electrical properties and superior corrosion resistance. Several excellent reviews are now available covering various aspects of this fast growing area of research (Anantharaman 1979; Cahn 1980; Luborsky 1979; Spaepen 1978). We shall confine ourselves to the most common methads for the production of these glasses and the need for experimentation in space for further development of these materials.

Among the methods for the production of metallic glasses may be mentioned the piston-and-anvil technique, melt spinning and melt extraction. In the first of these techniques, a molten drop of liquid metal is squeezed between a stationary anvil and a fast moving piston to yield a nearly circular disc of about $5-8 \mathrm{~mm}$ diameter and a thickness of about $40 \mu$. The exact time of release of the springloaded piston is determined with the aid of a photocell arrangement (figure 1). In the melt spinning process (figure 2) a jet of molten metal is ejected by a small gas pressure and is made to impinge on a rapidly rotating disc. It is thus converted into a tape of $1-150 \mathrm{~mm}$ width, $10-30 \mu$ thickness and the length of the tape can often be several hundreds of meters. Several factors, such as, melt superheat, the disc surface condition, the rate of revolution of the disc, orifice dimensions for jet formation, the angle of incidence of the melt, ejection pressure, etc., contral the thickness and the width of the ribbon obtained. In the melt extraction pracess, a ratating copper disc with a suitably designed groave- on its surface skims the surface of a molten metal bath and ejects fine wires of metallic glass.

Glass forming allays are often selected by determining their melting points vis-a-vis the melting temperatures of the constituent elements. In a given alloy system, the range of compositions within which the glass forms is often associated with the occurrerce of an eutectic or atleast a relatively low freezing temperature in relation to the melting points of constituent elements. Bulk of the glass forming metallic systems are either late transition metal-metalloid combinations or alloys of one transition metal with another. A few normal metal alloys and actinidetransition metal alloys have also been found to yield glasses. Most of the early transition metals and rare earths contain dissolved oxygen and vapour deposition experiments suggest that the formation of glasses in these systems is aided by the axygen already present in the alloy (Bennett and Wright 1972). The stability of the glasses so formed has also been found to be better as seen by an increase in the crystallisation temperature. Palk et al (1978) carried out experiments to test the applicability of these abservations to glasses produced directly from the melt. They nated that the glass transition temperature of an equiatomic $\mathrm{Zr}-\mathrm{Cu}$ glass, with about 2 at.\% axygen addition, is enhanced by $21 \mathrm{~K}$ with an attendant decrease in its crystallisation temperature. Since the difference between the crystal- 


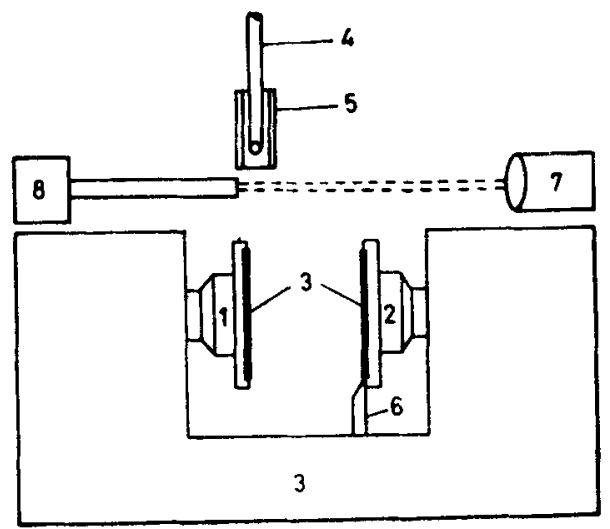
1. Fixed anvil
5. Susceptor
2. Fast-moving piston
6. Finger
3. Copper discs
7. Light source
4. Silice tube
8. Photo cell

Figure 1. The piston-and-anvil technique for the production of metallic glasses (Duwez, 1967)

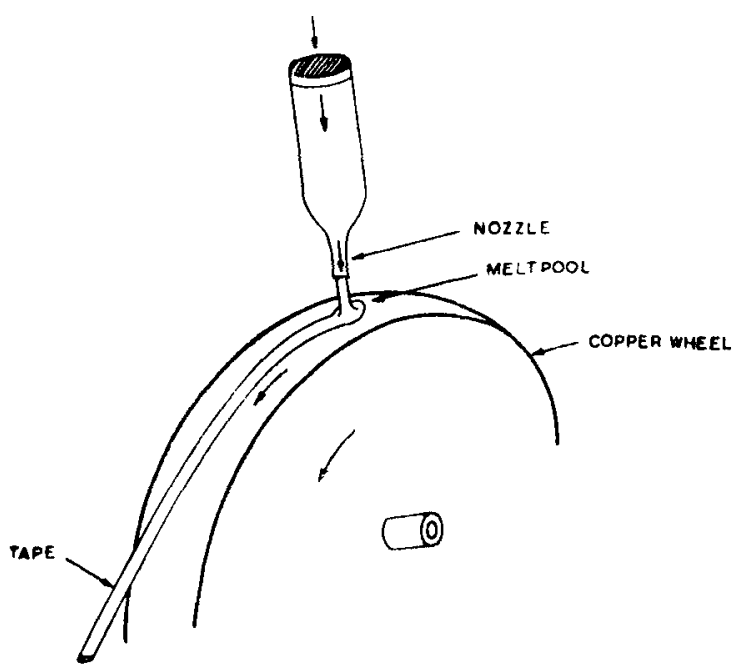

Figure 2. Principle of molt spinning of liquid metals and alloys.

lisation and glass transition temperatures is a measure of the ease of formation of the glass, it was argued that an addition of oxygen makes glass formation more difficult. The high levels of vacuum attainable in space should permit degassing and reduction in the levels of axygen in the melt and should, therefore, help in the formation of metallic glasses. True glass transition temperatures, unaltered by axygen, can thus be obtained in such experiments. 
The thearetical cooling rates required for glass formation are very high in most cases and actual glass formation is further restricted by the presence of heterogeneous impurities which could act as effective nucleation sites for crystallisation (Ramachandrarao 1977). The possibility of containerless melting under high vacuum in space enables the elimination of a large fraction of heterogeneous sites and should in principle make glass formation easier. Further, the passibility of accoustic positioning of a weightless mass of liquid can be profitably exploited in the production of large-sized metallic glass samples. While the need for a high critical cooling rate imposes unavaidable restrictions on the thickness of a metallic glass, use of pasitioning techniques in conjunction with the piston-and-anvil technique could greatly aid the preparation of metallic glass sheets with a large surface area. Such products will be of immense value in the manufacture of low loss transformer cores. Thus experiments on metallic glasses in space should prove to be richly rewarding.

\section{Acknowledgements}

The author is grateful to Professors S Ramaseshan and S Ranganathan for help and encouragement during the preparation of the manuscript.

\section{References}

Ahlborn H 1977 Hans. Tech., Essen, Vortragsveroeff. 391103

Anantharaman T R 1979 Trans. Indian Inst. Metals 3212

Bennett M R and Wright J G 1972 Phys. Status Solidi A13 135

Cahn R W 1980 Contemp. Phys. 2143

Chang C E and Wilcox W R 1975 J. Cryst. Growth 288

Deutscher K 1976 Proc. Second European Symp. on Materials Processing in space, Frascati, ESA-SP 114 p. 401

Duwez P 1967 Trans. Am. Soc. Metals 60607

Herr K, Barklage-Hilgefort H J and Frischat G H 1979 Proc. III European Symp. on Materials Science in Space, Grenoble, ESA-SP-142, p. 263

Kaye S 1974 J. Vac. Sci. Technol. 111114

Kohler H W 1978 Schweiz Alum. Rundsch 28151

Langlois W E 1979 J. Cryst. Growth 46743

Langlois W E $1980 \mathrm{~J}$. Cryst. Growth 4825

Luborsky F E 1979 Ferromagnetic materials (ed.) E R Wohlfahrt (Amsterdam : North Holland)

Polk D E, Dube C E and Giessan B C 1978 Rapidly quenched metals III. (ed.) B Cantor (London : Metals Soc.) 1220

Ramachandra Rao P, Cantor B and Cahn R W 1977 J. Mater. Sci. 122488

Sahm P R 1977 Haus Tech., Essen, Vortragsveroff 39133

Siebert G 1978a Prog. Cryst. Charact. 195

Siebex G 1978b Proc. R. Soc. London A361 131

Spaepen F 1980 Rapidly quenched metals III. (ed.) B Cantor (London : Metals Soc.) 2253

Takahashi S 1975 Bull. Jpn. Inst. Met. 14747

Walter H U 1977 Hans Tech., Essen, Vortragsveroff 39141 